\title{
Influencia de diferentes sistemas de profilaxis en la adhesión de un sellador de fisuras al esmalte

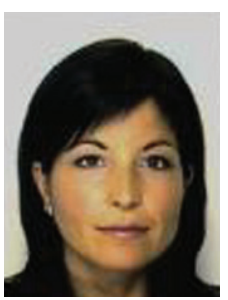 \\ Sol-Segarra, Eva
}

\author{
Influence of different prophylaxis sustems on the adhesion of a fissure sealant to the anamel
}

\section{Sol-Segarra, Eva* \\ Espasa-Suárez de Deza, Enrique** \\ Boj-Ọvesada, Juan Ramóñ}

\section{* Profesora asociada de Odontope- diatría \\ ** Profesor titular de Odontopediatría \\ ** Catedrático de Odontopediatría Odontopediatría, Facultad de Odon- tología, Universidad de Barcelona}

\section{Correspondencia}

Juan Ramón Boj Quesada

Facultad de Odontología

Pavelló de Govern $2^{\text {a }}$ Planta

Campus de Bellvitge

$\mathrm{C} /$. Feixa Llarga $\mathrm{s} / \mathrm{n}$

08907- L'Hospitalet de Llobregat.

Barcelona
Resumen: Introducción: La adhesión de las resinas dentales al esmalte no depende solamente del grabado ácido del esmalte. Otros factores, como el pulido de la superficie del esmalte pueden influir en la fuerza de adhesión de las resinas. El objetivo de este estudio es evaluar la adhesión de un sellador de fisuras fotopolimerizable tras la utilización previa de distintos métodos de profilaxis y dos diferentes tiempos de grabado. Material y método: Ochenta terceros molares extraídos quirúrgicamente se seccionaron en dos mitades (vestibular y lingual). Los dientes se dividieron en ocho grupos de 20 muestras cada uno, de acuerdo con los métodos de profilaxis: polvo de piedra pómez, pasta fluorada, espray de bicarbonato y control (sin profilaxis); y dos tiempos de grabado: 15 y 30 segundos. Se midió la fuerza de adhesión mediante una prueba de tracción en una máquina de ensayos universal INSTRON. Resultados y conclusión: Se utilizó el test estadístico ANOVA, detectándose diferencias significativas en la resistencia a la tracción; el posterior test de Dunnett encontró estas diferencias entre los grupos control y de profilaxis con piedra pómez para 15 y 30 segundos de grabado respecto de los grupos tratados con pasta fluorada y espray de bicarbonato, para los mismos tiempos de grabado $(p<0,05)$, siendo esta diferencia mayor en el grupo de espray con bicarbonato.

Palabras clave: Sellador de fosas y fisuras, Métodos de profilaxis, Grabado ácido, Resistencia a la adhesión.

Abstract: The adhesion of dental resins to the enamel not only depends on the acid etching, the prophylaxis methods can influence the bond strength. The objective of this study was to evaluate the adhesion of a light-cured sealant using previously different prophylaxis methods and two different etching times, thirty and fifteen seconds: Eighty, third molars surgically extracted were sectioned into two halves (buccal and lingual). The teeth were divided into 8 groups of 20 samples each, according to prophylaxis type: pumice powder, fluoridated paste, sodium bicarbonate spray, and control (no prophylaxis); and etching time: 30 and 15 seconds. Tensile bond strengths were studied using a universal Instron testing machine: A statistical ANOVA test was used, significant differences on the tensile strength test were detected; post-hoc Dunnett test founded these differences between control and pumice powder prophylaxis groups and the other two groups (fluoridated paste and sodium bicarbonate spray groups) for 15 and 30 seconds acid etching time $(p<0,05)$. Bond strength results were higher in the sodium bicarbonate group.

Key words: Pit and fissure sealant, Prophylaxis methods, Acid etching, Bond strength.

\begin{tabular}{ccc}
\hline Fecha recepción & Fecha última revisión & Fecha aceptación \\
$04-6-2003$ & $23-3-2004$ & $30-7-2004$ \\
\hline
\end{tabular}

BIBLID [1138-123X (2005)10:2; marzo-abril 125-240]

Sol-Segarra E, Espasa-Suárez de Deza E, Boj-Quesada JR. Influencia de diferentes sistemas de profilaxis en la adhesión de un sellador de fisuras al esmalte. RCOE 2005;10(2):177-182 


\section{Introducción}

En las últimas décadas la prevalencia de caries ha disminuido en los niños de los países desarrollados. Sin embargo, el mayor porcentaje de caries se sigue detectando en las fosas y fisuras, a pesar de la prevención con flúor, ya que se hallan menos protegidas por la acción de éste que las superficies lisas del diente $e^{1-5^{* *}}$.

El método que se ha revelado más efectivo en la prevención de caries oclusal es la utilización de selladores de fosas y fisuras; la mayor parte de estos materiales están basados en las resinas Bis-GMA $A^{6,7}$. La adhesión de estas resinas dentales al esmalte depende de la aplicación de un ácido que grabe el esmalte tal como describió Buonocore en 1955, pero también hay otros factores que juegan un papel importante como son el pulido de la superficie previamente al grabado, el tiempo de aplicación del ácido, la concentración y el tipo de ácido.

En cuanto al tiempo de grabado, varios autores recomiendan reducir el tiempo ya que no se observan diferencias en la adhesión de los selladores $^{8^{*}, 9}$.

Por profilaxis del esmalte entendemos la utilización, previa al grabado ácido, de métodos que eliminan la placa y los detritus orgánicos sin destruir la capa más superficial del mismo.

Los depósitos de la superficie dental pueden eliminarse por varios métodos: copas o cepillos rotatorios con pastas abrasivas, o bien puntas ultrasónicas. Todos estos métodos se basan en el principio de adaptar cristales abrasivos o impulsos ultrasónicos a la superficie que debe pulirse. Debido al contorno de la superficie dental,

\begin{tabular}{|ccc|}
\hline \multicolumn{3}{|c|}{ Tabla l. DistribuCí̃n de lOS gГIDOS } \\
\hline PROFILAXIS PREVIA & TIEMPO GRABADO & AC. FOSFORICO 37\% \\
\hline GRUPO (C-15) & NO & $15 \mathrm{seg}$. \\
\hline GRUPO (C-30) & NO & $30 \mathrm{seg}$. \\
\hline GRUPO (P-15) & Piedra pómez & $15 \mathrm{seg}$. \\
\hline GRUPO (P-30) & Piedra pómez & $30 \mathrm{seg}$. \\
\hline GRUPO (F-15) & Pasta fluorada & $15 \mathrm{seg}$. \\
\hline GRUPO (F-30) & Pasta fluorada & $30 \mathrm{seg}$. \\
\hline GRUPO (B-15) & Spray bicarbonato & $15 \mathrm{seg}$. \\
\hline GRUPO (B-30) & Spray bicarbonato & $30 \mathrm{seg}$. \\
\hline
\end{tabular}

la adaptación de estos instrumentos a la superficie de fosas y fisuras resulta prácticamente imposible. El método más tradicional consiste en una mezcla de polvo de piedra pómez y agua, pero se ha observado que utilizando piedra pómez pueden quedar restos de la misma que interfieran en el proceso del grabado ácido ${ }^{10-14^{* *}}$.

Durante tiempo, se ha recomendado no utilizar pastas con flúor o aceite puesto que se suponía que la presencia de estos componentes interfería en el grabado, pero no se han hallado evidencias concluyentes de que esto ocurra así1 ${ }^{13}$.

En 1977 se introdujo en odontología el pulido con partículas abrasivas. El primer aparato, Prophy-jet, fue patentado por Dentsply. Desde ese momento se han manufacturado diversos aparatos de este tipo como el Jet-polisher (Young Dental Mfg. St Louis, Mo. EUA), Stainbuster (Salem Dental Mfg, Salem, Om. EUA), Plaque Sweep (Dentsply/Equipment Div. York, Pa. EUA), etc. Este tipo de aparatos de pulido por aire actúan expulsando, sobre la superfície dental, un chorro controlado de partículas más o menos abrasivas (óxido de aluminio, sílice o bicarbonato de sodio) muy pequeñas, suspendidas en una aspersión de agua. Este sistema ha demostrado ser efectivo en la eliminación de la placa y las tinciones de la superficie denta|15-17.

El objetivo de este estudio es investigar la influencia de la profilaxis mediante espray de bicarbonato, previa al grabado ácido, en la fuerza de adhesión al esmalte de un sellador de fisuras de resina fotopolimerizable, mediante un ensayo de tracción, comparándola con otros métodos de profilaxis. También comprobaremos si el tiempo de aplicación del ácido ortofosfórico puede ser variable sin que por ello se afecten las fuerzas de enlace.

\section{Material y método}

Se recogieron 80 terceros molares incluidos, extraidos quirúrgicamente y que tuvieran la corona intacta. Se conservaron, durante tres meses de media hasta su estudio, en agua destilada. Se desechó la porción radicular y con una fresa diamantada en filo de cuchillo se seccionaron las coronas en dirección mesiodistal. Para el estudio se utilizaron las superficies vestibulares y linguales de las cuales se eliminó todo el esmalte excepto un círculo de $3 \mathrm{~mm}$ de diámetro. Los molares se 


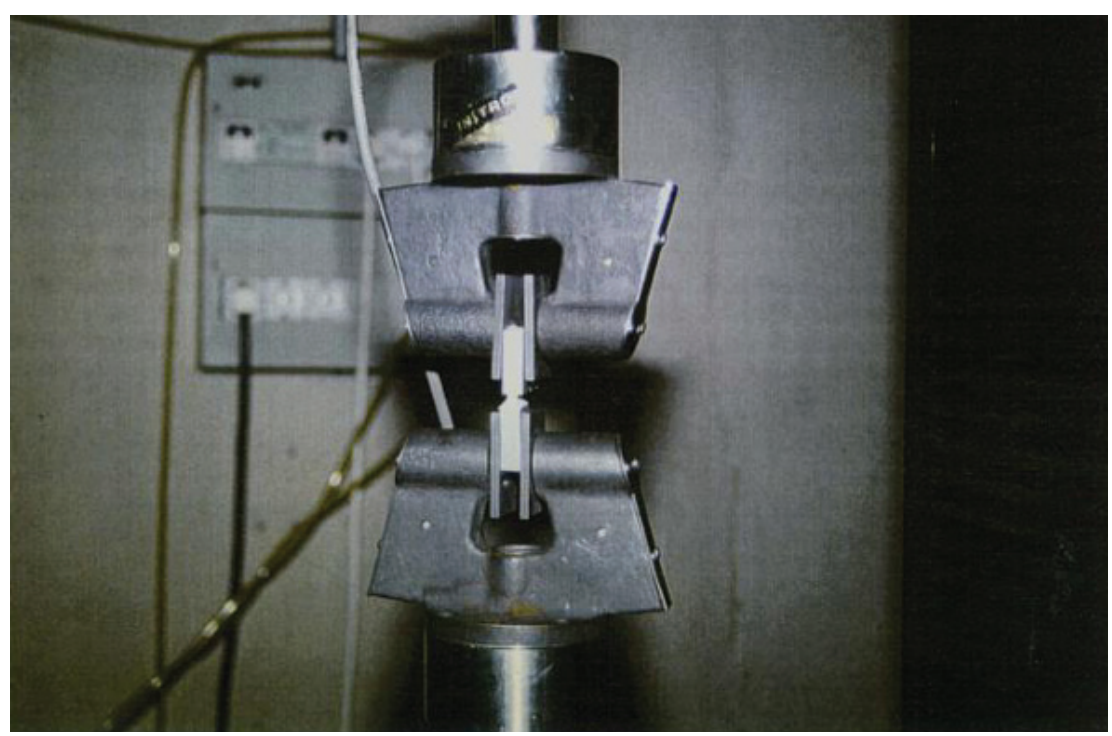

Figura 1. Bloques alineados en la máquina Instron.

introdujeron en yeso piedra dentro de un perfil de aluminio de medidas $1 \times 2 \times 2 \mathrm{~cm}$.

Las muestras se repartieron aleatoriamente formando ocho grupos de 20 superficies según la variable a estudiar (tabla 1).

Se prepararon unos bloques de resina autopolimerizable Formatray ${ }^{\circledR}$ a los que se les practicó una cavidad retentiva circular de $3 \mathrm{~mm}$ de diámetro y $5 \mathrm{~mm}$ de profundidad que se rellenó de composite $z-100 \AA$, hasta formar un cilindro de $3 \mathrm{~mm}$ de diámetro y que sobresalía $2 \mathrm{~mm}$ del bloque de resina.

Una vez preparados los dos tipos de bloques se montaron en una máquina de ensayos electromecánica INSTRON serie 4500 con mordazas neumáticas. En la abrazadera superior se colocó el bloque conteniendo el cilindro de composite y en la inferior el bloque conteniendo el diente pulido y grabado. Se aplicó una presión de cierre de 4 atmósferas para que no tuvieran lugar deslizamientos de las muestras sobre las mordazas. Se alinearon las muestras y se aproximaron a una velocidad de $2 \mathrm{~mm} /$ minutos hasta contactar el bloque superior con el sellador que previamente se había colocado sobre la superficie de esmalte. Al entrar en contacto los dos bloques solía derramarse algo de sellador que se absorbía con un trocito de papel secante. Después se fotopolimerizó el sellador, a una distancia de 3 $\mathrm{mm}$, en tres puntos diferentes durante 20 segundos en cada punto (fig. 1)

Se procedió al ensayo con la INSTRON poniendo en funcionamiento un mecanismo de tracción a una velocidad de $1 \mathrm{~mm} /$ minuto y una velocidad de adquisición de datos de 5 puntos/segundo hasta que la muestra rompiera y se separara el sellador del esmalte.

Para cada serie o grupo de tratamiento se halló la media y la desviación stándard respecto a la fuerza máxima, el desplazamiento a la fuerza máxima, la fuerza en el momento de la fractura y el desplazamiento en el momento de la fractura.

Después de realizar los ensayos de tracción, observamos las superficies de

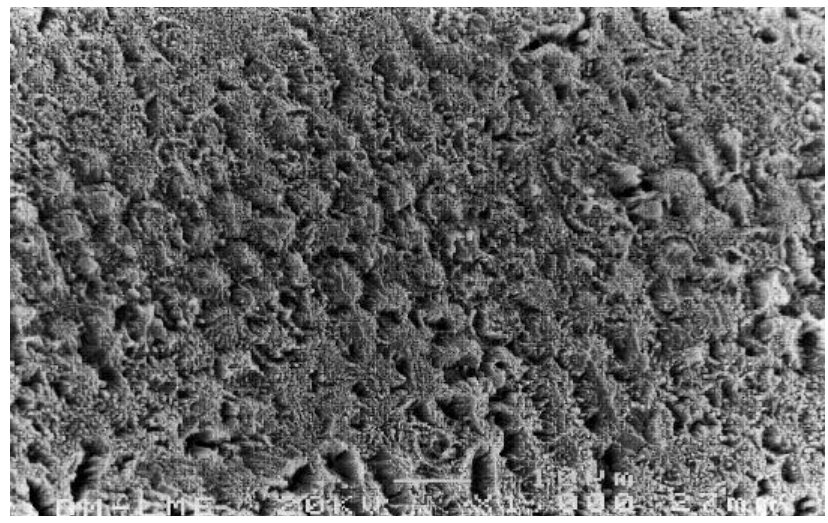

Figura 2. Vista al M.E.B. de una fractura (grupo B-15)

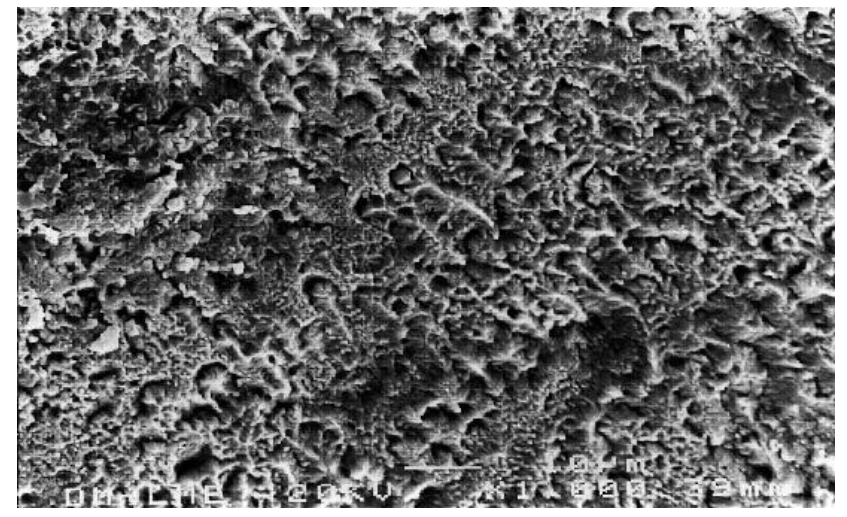

Figura 3. Vista al M.E.B. de una fractura (grupo C-3) 
fractura mediante microscopio electrónico JEOL 6400 a una diferencia de potencial de $20 \mathrm{Kv}$. Para ello fue preciso previamente someter las muestras al tratamiento de espolvoreado de oro al vacío durante 24 horas. Se fotografiaron las superficies de fractura (figs. 2 y 3 ).

\section{Resultados}

En la tabla 2 se han resumido los resultados obtenidos para cada grupo de estudio (fuerza media de adhesión en Mpa, desviación estándard y rango).

Se ha llevado a cabo un análisis de la varianza (ANOVA) de los resultados obtenidos para las distintas series ensayadas. Respecto a los tiempos de grabado ácido aplicados (15 y 30 segundos) hemos hallado que no influyen significativamente en la tensión máxima alcanzada para cada una de las series estudiadas, puesto que todos los valores de $p$ son mayores de 0,05 (nivel de significación empleado en todos los ensayos).

En relación con los métodos de profilaxis estudiados, al aplicar el test de comparación múltiple de medias de Dunnett, se observa que existen diferencias significativas y estas están en las series $F(p=0,037)$ y $B(p=0,004)$ donde se obtiene un aumento de la tensión máxima respecto a los grupos $P$ y control. En la serie $P$ no se observa una diferencia significativa respecto al control, ya que el valor de $p$ es superior a 0,05.

Además del estudio estadístico sobre fuerzas de adhesión también hemos realizado un estudio descriptivo, mediante microscopía electrónica, de las fracturas conseguidas con la

\begin{tabular}{|c|c|c|c|c|}
\hline \multicolumn{5}{|c|}{$\begin{array}{l}\text { Tabla e. Resultados obtenidos para cad } \\
\text { [Mpa:Megapascals] }\end{array}$} \\
\hline GRUPOS & $\begin{array}{c}\text { TIEMPO } \\
\text { GRABADO } \\
\end{array}$ & $\begin{array}{c}\text { FUERZA } \\
\text { (MPA) }\end{array}$ & $\begin{array}{l}\text { DESVIACION } \\
\text { STANDARD }\end{array}$ & RANGO \\
\hline (c)-15 & $15^{\prime \prime}$ & 3,2780 & 0,7149 & $2,0331-4,9134$ \\
\hline (c) -30 & $30 "$ & 3,4395 & 0,5817 & $2,5359-4,3847$ \\
\hline $\mathrm{P}-15$ & $15^{\prime \prime}$ & 3,9886 & 1,7256 & $1,9805-4,6826$ \\
\hline$P-30$ & $30 "$ & 3,4936 & 1,0749 & $2,0937-4,6826$ \\
\hline $\mathrm{F}-15$ & 15" & $4,1442^{*}$ & 1,1178 & $2,4757-6,0395$ \\
\hline$F-30$ & $30 "$ & $4,5827^{*}$ & 0,7355 & $3,1265-5,7012$ \\
\hline B-15 & $15^{\prime \prime}$ & $4,6393^{\star}$ & 1,1881 & $2,8860-7,3847$ \\
\hline B-30 & $30^{\prime \prime}$ & $4,7241^{*}$ & 1,2022 & $3,0274-6,8047$ \\
\hline
\end{tabular}

INSTRON. Hemos observado que en todos los grupos el patrón de fractura predominante ha sido el de tipo cohesivo en el sellador.

\section{Discusión}

Previamente al grabado ácido del esmalte hemos realizado una profilaxis con distintos métodos para intentar aumentar las fuerzas de unión entre el esmalte y el sellador de fisuras. Sin embargo, la mayoría de estudios que hemos revisado realizan un aplanamiento de la cara vestibular con la idea de aplicar fuerzas lo más perpendiculares posible sobre la superficie a examinar. Este aplanamiento se lleva a cabo mediante discos de papel abrasivo y conlleva a su vez un decapado o eliminación de la capa más superficial del esmalte. Al practicar este decapado se elimina la capa más aprismática del esmalte y por tanto se consigue una superficie capaz de interaccionar mejor con el grabado ácido, pudiéndose obtener mayores fuerzas de enlace con las resinas, ya sean de composite o selladores. Sin embargo, cuando se va a aplicar un sellador de fisuras no se realiza este pulido tan agresivo. Este procedimiento con cierto carácter invasivo se correspondería más con la técnica denominada ameloplastia que es ligeramente distinta al sellado de fisuras propiamente dicho. Por ello hemos creido conveniente no abrasionar con discos la superficie más externa aún a costa de medir fuerzas de resistencia a la tracción no totalmente perpendiculares y obtener resultados más bajos que la mayoría de estudios ${ }^{11-14^{* *}}$

El tiempo estándar que se ha utilizado clásicamente para el grabado ácido del esmalte ha sido de 60 segundos. Recientemente varios trabajos han indicado que una reducción en el tiempo de grabado disminuye la pérdida de esmalte pero sin afectar los resultados clínicos de adhesión entre los selladores $u$ otros tipos de resinas que se utilizan en odontología conservadora y el esmalte. 
Nosotros también hemos hallado que no existen diferencias significativas en cuanto a la fuerza de adhesión del sellador de fisuras utilizado, aplicando fuerzas de tracción, al variar el tiempo de grabado entre 15 y 30 segundos (para 15 segundos de grabado hemos obtenido una tensión media de 4,0212 MPa y para 30 segundos la tensión es de 4,0599 MPa) $(p>0,05)$.

Respecto a los distintos métodos de profilaxis coincidimos con los estudios que afirman que no existen diferencias significativas, en cuanto a fuerzas de adhesión, entre realizar la profilaxis previa del esmalte con pastas fluoradas o no, ya que nosotros hemos obtenido resultados similares entre los grupos tratados con pasta fluorada (la tensión media entre F-15 y F-30 es de 4,342 MPa) y los grupos tratados con pasta a base de piedra pómez (la tensión media entre P-15 y P-30 es de 3,730 MPa); incluso obser- vamos una mayor resistencia a la tracción en el grupo tratado con pasta fluorada. Aboush y collis tampoco hallaron diferencias significativas con diferentes pastas que contienen flúor o aceite. También Ray Bogert y GarcíaCodoy ${ }^{18}$ realizaron un estudio de cizallamiento con pastas con o sin flúor, que además compararon con la profilaxis clásica con pasta de piedra pómez y con un grupo control que sólo se limpió con agua; observaron que ninguno de estos métodos influía en las fuerzas de adhesión de selladores y que, por lo tanto, el grabado ácido por si solo ya era suficiente para eliminar la película adquirida del esmalte.

Coincidimos con el trabajo de Brown y $\mathrm{col}^{14^{* *}}$ que hallan mejores resultados con la utilización del pulido con aire, bien sea con partículas de óxido de aluminio o de bicarbonato sódico, como complemento del grabado ácido.
En todos los grupos el tipo predominante de fractura obtenido fue cohesivo en el sellador sin influir el método de profilaxis realizado, lo que indicaría por tanto una mayor persistencia de tags de resina unida a la superficie del esmalte y en consecuencia una buena calidad de la unión esmalte-sellador en todos los casos estudiados.

Encontramos de forma significativa una mayor resistencia a la tracción en dos de los métodos de profilaxis empleados (pasta de profilaxis fluorada y espray de bicarbonato $(p<0,05)$, respecto al grupo control y al grupo tratado con pasta a base de polvo de piedra pómez $(p>0,05)$.

Entre los dos métodos de profilaxis que observamos mayor resistencia a la tracción, los valores más altos se obtuvieron en el grupo en que se empleó la profilaxis con espray de bicarbonato, pero esta diferencia no fue significativa.

\section{Bibliografla recomendada}

Para profundizar en la lectura de este tema, el/los autor/es considera/an interesantes los artículos que aparecen señalados del siguiente modo: *de interés **de especial interés.

1. Simonsen RJ. Retention and effectiveness of dental sealant after 15 years. J Am Dent Assoc 1991;122:34-42.

2. Estrela-Sanchís F, Almerich Silla JM. Preparación de la superficie oclusal antes de la aplicación del sellado de fisuras: estudio in vitro. RCOE 1997:2:95-106.

3. Vehkalati MM, Solavaara L, Rytöma I. An eight year follw-up of the occlusal surfaces of first permanent molars. J Dent Res 1991;70:1064-7.

4. Stahl JW, Katz RV. Occlusal dental caries incidence and implications for sealant programs in a US college student population. J Public Health Dent 1993;53:212-8.
5. Bader JD, Shugas DA, Bonito AJ. A systematic review of selected caries prevention and management methods. Community Dent Oral Epidemiol 2001;29:399-411.

$6^{* *}$. Simonsen RJ. Pit and fissure sealant: review of the literature. Pediatr Dent 2002;24:393-414. Una revisión exhaustiva de la literatura sobre los selladores de fisuras, desde 1971 hasta octubre de 2001. Muy interesante para situar al lector en los diferentes aspectos que están sujetos a estudio en el campo de los selladores, pues abarca todos los temas relacionados con su aplicación y sus indicaciones.

7. Donly K. Sealants: where we have been; whe- re we are going. Gen Dent 2002;50:438-40.

8*. Barkmeier WW, Gwinnett AJ, Shaffer SE Effect of reduced acid concentration and etching time on bond strength and enamel morphology. J Clin Orthod 1997;21:395-8.

En este trabajo los autores examinan el efecto de la reducción de la concentración de ácido fosfórico y del tiempo de grabado sobre la fuerza de adhesión aplicando fuerzas de cizallamiento. La reducción del tiempo de grabado es una de las variables también estudiada en nuestro trabajo.

9. Legler LR, Retief DH, Bradley EL, Denys FR, Sadowsky PL. Effects of phosphoric acid con- 
centration and etch duration on the shear bond strength of an orthodontic bonding resin to enamel. Am J Orthod Dentofacial Orthop 1989;3:485-92,

10. Suárez-Quintanilla JM, Suárez-Quintanilla D. Estudio del grabado ácido en las fisuras mediante microscopía electrónica de barrido. Rev Esp Odonto-Estomatol 1987;35:467-74.

11. Scott L, Greer D. The effect of an air-polishing device on sealant bond strength. J Proth Dent 1987;58:384-7.

12. Huennekeus S, Daniel S, Bayne S. Effects of air-polishing on the abrasion of occlusal sealants. Quintessence Int 1991;22:581-5.

13. Aboush YEY, Tareen A, Elderton RJ. Resin to enamel bonds: effects of cleaning the enamel surface with prophylaxis pastes containing fluoride or oil. Br Dent J 1991;171:207-9.

$14 * *$. Brown J, Barkmeier WA. A comparison of six enamel treatment procedures for sealant bonding. Pediatr Dent 1996;18:29-31.

Este trabajo evalúa la fuerza de adhesión de un sellador al esmalte, mediante la resistencia al cizallamiento, comparando distintos tratamientos de la superficie del esmalte, los valores de fuerza de adhesión más altos corresponden a los grupos que habían recibido un pulido previo con aire, bien con partículas de óxido de aluminio o bien con bicarbonato y posterior grabado; sin embargo estos resultados no son estadísticamen- te significativos en relación con los del grupo control que sólo había recibido grabado ácido.

15. Strand GV, Raadal M. The efficiency of cleaning fissures with an air-polishing instrument. Acta Odontol Scan 1988;46:113-7.

16. Konturi Narhi V, Markkanen S. Effects of airpolishing on dental plaque removal and hard tisues as evaluated by scanning electrón microscopy. J Periodontol 1990;61:334-8.

17. Porth R.N. New concepts in air abrasion: Dent Today 1998; 17:66,68,70-1.

18. Ray-Bogert T, García-Godoy F. Effect of prophylaxis agents on the shear bond strength of a fissure sealant. Pediatr Dent 1992;14:50-1. 